\title{
THE MENTAL HEALTH OUTCOMES AND ASSESSMENT TOOLS TRAINING PROJECT: CREATING THE FOUNDATION FOR IMPROVED QUALITY OF CARE
}

\author{
Jennifer Chipps and Beverley Raphael \\ Centre for Mental Health \\ NSW Department of Health \\ Tim Coombs \\ New South Wales Institute of Psychiatry
}

The NSW Mental Health Outcomes and Assessment Tools Training Project (MH-OAT) is a collaborative and consumer-centred project that aims to strengthen the assessment skills of the mental health care workforce. MHOAT does this through training that supports the introduction of a process of standard documentation of clinical practice along with measures of outcomes and potential 'casemix' (a method of describing the different types of patients treated by the health system, which recognises that different patients require different levels of resources). This article describes the rationale for $\mathrm{MH}-$ OAT, its implementation, and some of the issues identified during implementation.

\section{BACKGROUND}

The Caring for Mental Health framework (1998) outlined the strategic direction for mental health services in New South Wales. ${ }^{1}$ This framework included:

- standard documentation for health assessment and triage activities;

- measures of health outcomes;

- national standards for mental health services.

In parallel with this state framework, the introduction of standard measures of outcomes and potential casemix has been agreed to by all states and territories, as part of the information development strategy of the Second National Mental Health Plan. ${ }^{2,3}$

MH-OAT was developed to enable the goals of the state framework and of the national strategy to be achieved. MH-OAT has three core components, all of which are collaborative and consumer-centred. The first, a training component, is aimed at strengthening the assessment skills of the mental health workforce. The second, standard modules of clinical documentation, is aimed at improving the quality of health assessment and care of mental health clients. Third, is the collection of standard measures of outcomes and casemix.

\section{IMPLEMENTATION OF MH-OAT}

The implementation of MH-OAT involved:

- a planning framework;

- a communication strategy;

- an education and training strategy;
- a data capture and reporting system;

- ongoing support.

Planning framework

To facilitate the implementation of MH-OAT, a steering committee was established, a coordinator appointed, and the following resources were developed:

- standard modules of clinical documentation for triage assessment, admission, review, care, and discharge from mental health care;

- a protocol for data collection that identifies the outcome measures to be collected at various points in the care of each consumer of mental health services.

Funding was secured to support the release of staff for training, and for the appointment of a coordinator to support the implementation process.

\section{Communication strategy}

It was essential to communicate the intention and purpose of MH-OAT to the mental health care workforce. Consequently, as a first step, awareness-raising workshops were held with managers in the area mental health services and with senior clinicians in mental health. These workshops outlined the local implementation process, which included the development of a business case, the appointment of a local MH-OAT coordinator in each area, and the creation of local implementation committees with representation from stakeholders.

The second step was to initiate consultation with stakeholders. As important stakeholders are consumers of mental health care, a Consumer Consultation Group was established. Other stakeholders included representatives from the Royal Australian and New Zealand College of Psychiatrists, the Australian and New Zealand College of Mental Health Nurses, the Divisions of General Practice, the NSW Nurses Association, Carers Groups, multicultural groups, tertiary institutions, and Aboriginal and Torres Straight Islander groups. This consultation process is ongoing.

Information was disseminated through regular updates by newsletters, and through the intranet and internet. The MH-OAT website allows both consumers and the mental health workforce to download all material related to $\mathrm{MH}$ OAT from training to progress reports on implementation.

\section{Education strategy}

It was estimated that the equivalent of approximately 4700 full-time clinical mental health staff would require training. Due to the large number, a 'Train the Trainer' model was selected and training materials were developed to support the facilitation of training small groups within 
the area health services. In total, 14 'Train the Trainer' workshops were held, and the participants in those workshops have now become trainers and resource people within the area health services.

The aim was that each clinician receive eight hours of training to support the clinical processes and documentation of standard modules for mental health triage assessment, care planning, and so forth; and four hours of training in the standard measures of health outcomes. As part of this training, each mental health clinician was required to observe an assessment and a case review, using the standard documentation and outcome measures.

\section{Data capture and reporting system}

A computer application supports the collection, storage, extraction, analysis, reporting, and feedback of the deidentified data on outcomes and casemix. Because the NSW Health strategic solution to the collection of data in the community, Community Health Information Management Enterprise (CHIME), was not available, an interim Access application was developed. This interim system, Service Contact Information-Mental Health Outcomes and Assessment Training (SCI-MH-OAT) was developed as the basis of recording and to provide feedback to clinicians.

\section{Ongoing support}

To ensure that MH-OAT continues to be implemented:

- coordinator positions have been funded at the local area level;

- $\quad$ support visits are being made to local staff in the area mental health services;

- training has been linked to ongoing staff orientation and university curricula;

- collaboration is occurring with the collection of a minimum data set, to both improve data collection and to monitor the progress of implementation.

\section{ISSUES IDENTIFIED DURING IMPLEMENTATION}

A number of issues were identified during the initial phases of implementation:

- the communication and reinforcement of core messages is essential. For example, when standard documentation is completed, this should not be seen as a structured interview with a client; instead, it should support the clinical process. The reinforcement of this message has been achieved through the development of a pamphlet for staff;
- the initial clinical documentation was too onerous. Initial feedback suggests duplication of information collection within the documents. The modules will be adapted in 2003;

- the level of provision of information technology in the area health services is being further developed;

- the computer literacy of staff is variable and additional training is required;

- concerns about the validity and reliability of the selected standard measures. The standard measures of outcomes and casemix used as part of the MH-OAT initiative are seen as a first step in the development of a culture of systematic outcome monitoring. To this end, it is acknowledged that the utility of the standard measures will require review and additional measures for specific purposes will need to be identified.

\section{CONCLUSION}

To date, about 95 per cent of mental health staff have been trained and about 60 per cent of area mental health services have commenced data collection. The implementation of MH-OAT has involved a significant change in work practice for staff and, at present, there are different levels of acceptance. However, there is a universal acceptance of the principles underpinning $\mathrm{MH}$ OAT. During consultation, it was clear that the mental health workforce wants both better mental health assessment and documentation for their clients, and that they support the development of the routine collection of health outcomes and casemix information to encourage a culture of evidence-based practice. How these principles are achieved will be part of the continued development of MH-OAT.

Further information about MH-OAT can be obtained from the NSW Department of Health website at www.health.nsw.gov.au/policy/cmh/mhoat, or by email from mhoat@doh.health.nsw.gov.au.

\section{REFERENCES}

1. Policy Division. Caring for Mental Health: A Framework for Mental Health Care in NSW. Sydney: NSW Department of Health, 1998.

2. Department of Health and Aged Care. Mental Health Information Development: National Information Priorities and Strategies under the Second National Mental Health Plan 1998-2003. Canberra: Commonwealth of Australia, 1999. 